University of Wollongong

Research Online

Australian Institute for Innovative Materials -

Papers

Australian Institute for Innovative Materials

$1-1-2017$

\title{
Activated carbon from the graphite with increased rate capability for the potassium ion battery
}

Zhixin Tai

University of Wollongong, zt525@uowmail.edu.au

Qing Zhang

University of Wollongong, qz964@uowmail.edu.au

Yajie Liu

University of Wollongong, yl327@uowmail.edu.au

Hua-Kun Liu

University of Wollongong, hua@uow.edu.au

Shi Xue Dou

University of Wollongong, shi@uow.edu.au

Follow this and additional works at: https://ro.uow.edu.au/aiimpapers

Part of the Engineering Commons, and the Physical Sciences and Mathematics Commons

Research Online is the open access institutional repository for the University of Wollongong. For further information contact the UOW Library: research-pubs@uow.edu.au 


\title{
Activated carbon from the graphite with increased rate capability for the potassium ion battery
}

\author{
Abstract \\ Activated carbon has been synthesized by a high-temperature annealing route using graphite as carbon \\ source and potassium hydroxide as the etching agent. Many nanosized carbon sheets formed on the \\ particles could be of benefit for rapid intercalation/de-intercalation of potassium ions. Moreover, the d- \\ spacing in the (100) crystal planes of the as-prepared active carbon is enlarged to $0.335 \mathrm{~nm}$, even some \\ formed carbon nanosheets can reach $0.358 \mathrm{~nm}$, and the diffusion coefficient of $\mathrm{K}$ ion is also improved by \\ 7 times as well. The as-prepared activated carbon electrode can deliver a high reversible capacity of 100 \\ mAh g i 1 after 100 cycles (at a high current density of $0.2 \mathrm{~A} \mathrm{~g} i 1$ ), and exhibits increased rate \\ performance. The results demonstrate that the as-prepared active carbon is a promising anode material \\ for the potassium ion battery, with high capacity, excellent rate performance, and great cycling stability.

\section{Disciplines} \\ Engineering | Physical Sciences and Mathematics

\section{Publication Details} \\ Tai, Z., Zhang, Q., Liu, Y., Liu, H. \& Dou, S. (2017). Activated carbon from the graphite with increased rate \\ capability for the potassium ion battery. Carbon, 123 54-61.
}




\title{
Activated carbon from the graphite with increased rate capability for the potassium ion battery
}

\author{
Zhixin Tai, ${ }^{1}$ Qing Zhang, ${ }^{1}$ Yajie Liu, Huakun Liu ${ }^{1}$ and Shixue Dou \\ Institute for Superconducting and Electronic Materials, Australian Institute for Innovative Materials, University of \\ Wollongong, Innovation Campus, North Wollongong, New South Wales 2500, Australia \\ ${ }^{1}$ These authors contributed equally to this work.
}

\begin{abstract}
Activated carbon has been synthesized by a high-temperature annealing route using graphite as carbon source and potassium hydroxide as the etching agent. Many nanosized carbon sheets formed on the particles could be of benefit for rapid intercalation/de-intercalation of potassium ions. Moreover, the $d$-spacing in the (100) crystal planes of the as-prepared active carbon is enlarged to $0.335 \mathrm{~nm}$, even some formed carbon nanosheets can reach $0.358 \mathrm{~nm}$, and the diffusion coefficient of $\mathrm{K}$ ion is also improved by 7 times as well. The as-prepared activated carbon electrode can deliver a high reversible capacity of $100 \mathrm{mAh} \mathrm{g}^{-1}$ after 100 cycles (at a high current density of $0.2 \mathrm{~A} \mathrm{~g} \mathrm{~g}^{-1}$ ), and exhibits increased rate performance. The results demonstrate that the as-prepared active carbon is a promising anode material for the potassium ion battery, with high capacity, excellent rate performance, and great cycling stability.
\end{abstract}

$1 *$ Corresponding author. Institute for Superconducting and Electronic Materials, Australian Institute for Innovative Materials, University of Wollongong, Innovation Campus,North Wollongong, New South Wales 2500, Australia. Tel:+61242214547 E-mail: hua_liu@uow.edu.au 


\section{Introduction}

The great challenge of electrochemical energy storage (EES) for not only electric vehicles, but also portable electronic devices (e.g., mobile phones, laptop computers) has driven researchers to endlessly look for more promising candidates for new energy storage systems. Among them, the alkali metals, particularly lithium (Li), have been brought into industrial utilization, and graphite has also been developed as the standard anode for the $\mathrm{Li}^{+}$battery due to its high reversible capacity, flat voltage, and low cost. Concerns about future shortages of lithium resources have shifted primary attention to the more abundant resources, such as sodium $(\mathrm{Na})$ and potassium $(\mathrm{K})$. As reported, graphite anode exhibits a higher theoretical capacity $\left(\sim 270 \mathrm{mAh} \mathrm{g}^{-1}\right)$ in potassium ion batteries (KIBs) than in sodium ion batteries (NIBs) $\left(\sim 35 \mathrm{mAh} \mathrm{g}^{-1}\right)$, indicating the KIBs may be a better candidate than SIBs for commercial graphite anode.[1]. Meanwhile, the potassium ion battery has attracted more and more attention recently due to the wide distribution of potassium and the higher redox potential of $\mathrm{K} / \mathrm{K}^{+}(-2.92 \mathrm{~V}$ vs. standard hydrogen electrode (SHE)) compared to that of $\mathrm{Na} / \mathrm{Na}^{+}(-2.71 \mathrm{~V}$ vs. SHE), which makes the potassium ion battery a potential alternative to lithium ion batteries.

Recently, several carbon-based materials have been introduced as anode electrodes for KIBs. After demonstrating a three-stage process for $\mathrm{K}^{+}$intercalation into graphite $(\mathrm{C}$ $\rightarrow \mathrm{KC}_{24} \rightarrow \mathrm{KC}_{16} \rightarrow \mathrm{KC}_{8}$ ), Ji and his co-workers found out that the high rate performance of graphite anode is suboptimal in a nonaqueous electrolyte, so they proposed a soft carbon as a high rate anode material in KIBs with a theoretical capacity of $273 \mathrm{mAh}$ $\mathrm{g}^{-1}$ [2]. And potassium shuttlecock mechanism between two insertion materials as potassium ions battery is advantageous for higher-voltage/-power rechargeable batteries due to the lowest potential and weakest solvation among $\mathrm{Li}^{+}, \mathrm{Na}^{+}, \mathrm{K}^{+}, \mathrm{Mg}^{2+}$, and $\mathrm{Ca}^{2+}$ ion carriers.[3] Furthermore, Ji et al. also reported hard carbon microspheres (HCS) that showed a high initial capacity of $262 \mathrm{mAh} \mathrm{g}^{-1}$, with $83 \%$ capacity retention over 100 cycles in KIBs. HCS presented a much better high rate performance in KIBs, compared to their behavior in NIBs, which can be ascribed to a higher diffusion coefficient of K-ions in the particular structure compared to Na-ions 
[4]. The three-stage process for $\mathrm{K}^{+}$intercalation into graphite was further confirmed in a reduced graphene oxide (RGO) film electrode, and a high-performance electrode was also demonstrated [5]. Those carbon-based materials have thus shown their great potential for KIB application. Among all the carbon-based materials, however, low-cost graphite as the commercial electrode material for the lithium ion battery displays limited electrochemical performance when it is directly used in $\mathrm{K}^{+}$batteries. Generally, the high ion diffusion resistance of graphite, resulting from its narrow interlayer spaces $(0.34 \mathrm{~nm})$ and the long diffusion pathways (several micrometers) for potassium ions is the major obstacle to the incorporation of potassium ions into a metal-carbon compound with the stoichiometry of $\mathrm{KC}_{8}$, which directly affects its rate capability $[2,5]$. Moreover, comparing the ionic radii, the $\mathrm{K}^{+}$ion $(1.33 \mathrm{~nm})$ is larger than the $\mathrm{Li}^{+}$ion $(0.59 \mathrm{~nm})$, so $\mathrm{K}^{+}$has much more difficulty in intercalating into graphite than $\mathrm{Li}^{+}$, which is also conducive to the reduced rate performance of graphite electrode in KIBs.

In order to facilitate the application of low-cost and commercially available graphite in potassium ion batteries with enhanced electrochemical performance in this work, activated carbon was fabricated by an etching method. After treatment of the graphite, their interplanar spacing was enlarged, and the ion diffusion coefficient during the electrochemical process was enhanced as well. These improvements to the activated carbon would play a significant role in increasing its rate performance as an anode for KIBs.

\section{Experimental}

\subsection{Methods}

High-purity (99.99\%) graphite (325 mesh) was used to prepare activated graphite. Typically, $1 \mathrm{~g}$ graphite was dispersed in $30 \mathrm{~g}$ water to create a $\sim 3 \mathrm{wt} \%$ dispersion. After stirring for $30 \mathrm{~min}, 1 \mathrm{~g} \mathrm{KOH}$ powder was put into the prepared graphite solution, and the mixture was kept under stirring for $2 \mathrm{~h}$ at room temperature. Then, the prepared graphite/KOH solution was moved into an air-circulating oven at $80{ }^{\circ} \mathrm{C}$ to evaporate the water. The resultant powder was then heated at $800{ }^{\circ} \mathrm{C}$ for $2 \mathrm{~h}$ in a tube 
furnace under flowing argon gas. Afterward, the synthesized powder was washed with $1 \mathrm{M} \mathrm{HCl}$ and distilled water several times to remove the residual $\mathrm{KOH}$. Then, the final product (denoted as $\mathrm{AC}-1$ for its ratio of $\mathrm{KOH}$ to graphite, 1:1) was collected by vacuum filtration and dried in a vacuum oven. Also, we made samples with 2:1 and 3:1 ratios of $\mathrm{KOH}$ to graphite, which are denoted as AC-2 and AC-3, respectively. The commercial graphite was denoted as AC-0.

\subsection{Characterization}

The microstructure of the activated graphite was investigated with a Raman system (Thermo Nicolet Almega XR Raman Microscope, with an excitation wavelength of $532 \mathrm{~nm}$ ). X-ray diffraction (XRD) patterns were collected with a Rigaku D/MAZX $2500 \mathrm{~V} / \mathrm{PC}$ with $\mathrm{Cu} \mathrm{K} \alpha$ radiation $(35 \mathrm{kV}, 20 \mathrm{~mA}, \lambda=1.5418 \AA)$. Scanning electron microscope (SEM) images were obtained with a JEOL JSM-7500 scanning electron microscope. High-resolution transmission electron microscope (HRTEM) images were obtained using a JEOL 2010 operating at $200 \mathrm{kV}$. Nitrogen adsorption isotherm measurements were performed on a Nova 1000 volumetric adsorption analyzer at $77 \mathrm{~K}$. The Brunauer-Emmett-Teller (BET) method was utilized to calculate the specific surface area of each sample.

\subsection{Electrochemical Tests}

The electrodes were composed of the activated graphite, carbon additive (Super-P), and polyvinylidene fluoride (PVDF) binder, with a mass ratio of 8:1:1. Here, $10 \%$ of super-P was absolutely not the indispensable for preparation of the electrode due to the good conductivity of the graphite. Because $8: 1: 1$ of the Active materials/Binder/Super-P is a classical ratio for the electrode, the addition of the $10 \%$ super-P will benefit the comparison with other results from other researchers. The slurry was cast onto $\mathrm{Cu}$ foil and dried at $120^{\circ} \mathrm{C}$ under vacuum for $12 \mathrm{~h}$. Coin cells (CR2032) were assembled with potassium foil as the counter/reference electrode, a glass fiber separator, and $0.8 \mathrm{M} \mathrm{KPF}_{6}$ in ethylene carbonate/diethyl carbonate (EC/DEC, 1:1 v/v) as the electrolyte in an argon-filled glove box. Charge/discharge measurements were carried out on a Neware battery test system (BTS-XWJ-6.44S-00052, Neware, Shenzhen, China) at different current densities 
between 0.01 and $2.00 \mathrm{~V}$ vs. $\mathrm{K} / \mathrm{K}^{+}$or $\mathrm{Na} / \mathrm{Na}^{+}$at room temperature. Electrochemical impedance spectroscopy (EIS) was conducted using a Biologic VPM3 electrochemical workstation.

\section{Results and discussion}

The morphologies of the commercial graphite and the synthesized activated carbon were characterized by SEM, as shown in Fig. 1. AC-0 possessed sheet-like particles and had a smooth surface, whereas, the morphology of samples from AC-1 to AC-3 shows a rough surface, and the particle size decreases from several micrometers to several nanometers with increasing $\mathrm{KOH}$, as shown in Fig. $1 \mathrm{~b}$ and c. On comparing the details of AC-2 and AC-3, the surface of AC-3, as shown in Fig. 1d, appeared less rough than in the case of the AC-2 because of the over-etching effect of $\mathrm{KOH}[6,7]$. The evolution of this process is illustrated in Fig. 1e. The results indicated that many large carbon sheets on the surface of the particles had been smashed into many small pieces by the activation. Meanwhile, more interface surface for potassium ion transmission was generated on the carbon layer. 

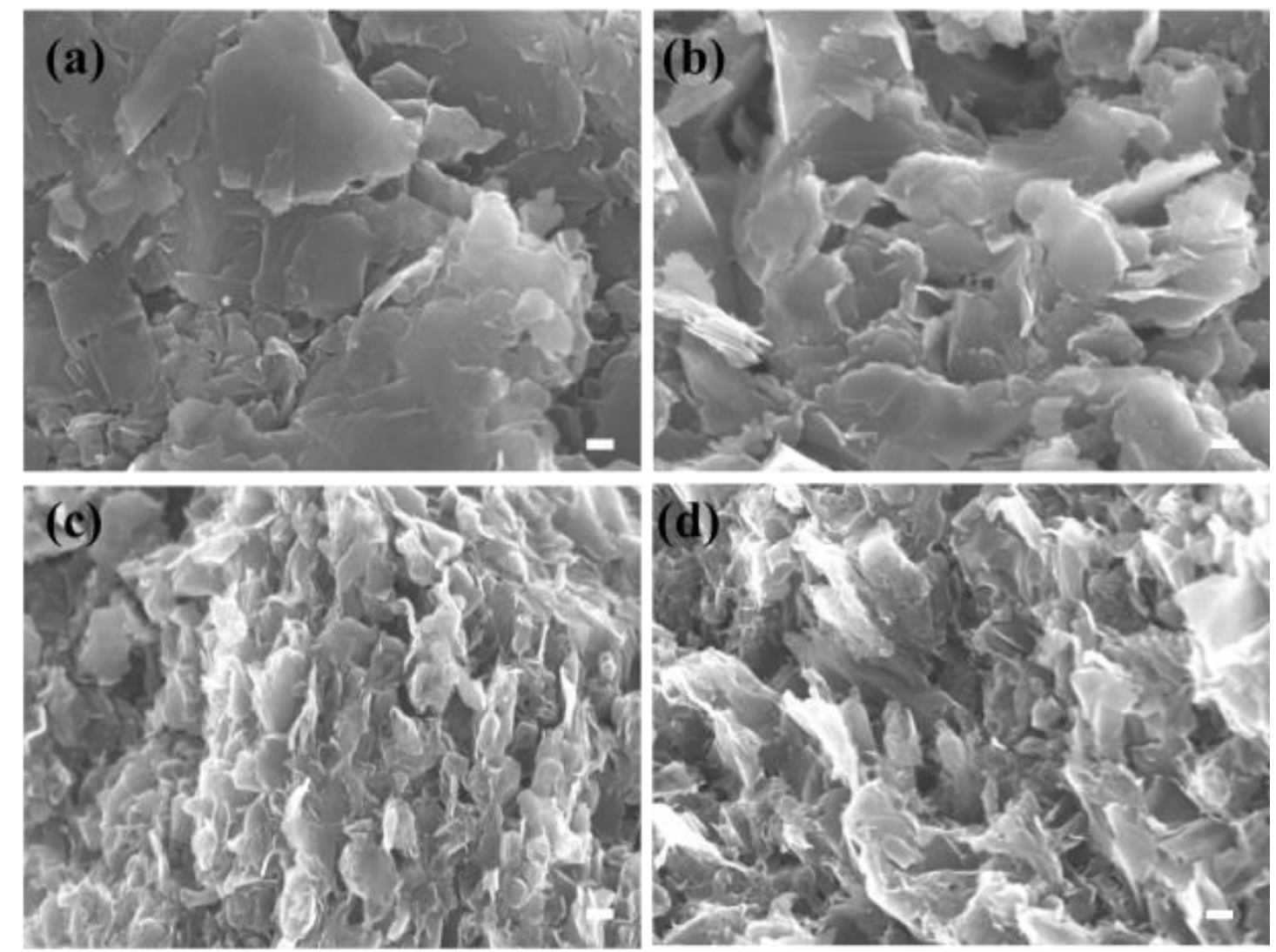

(e)
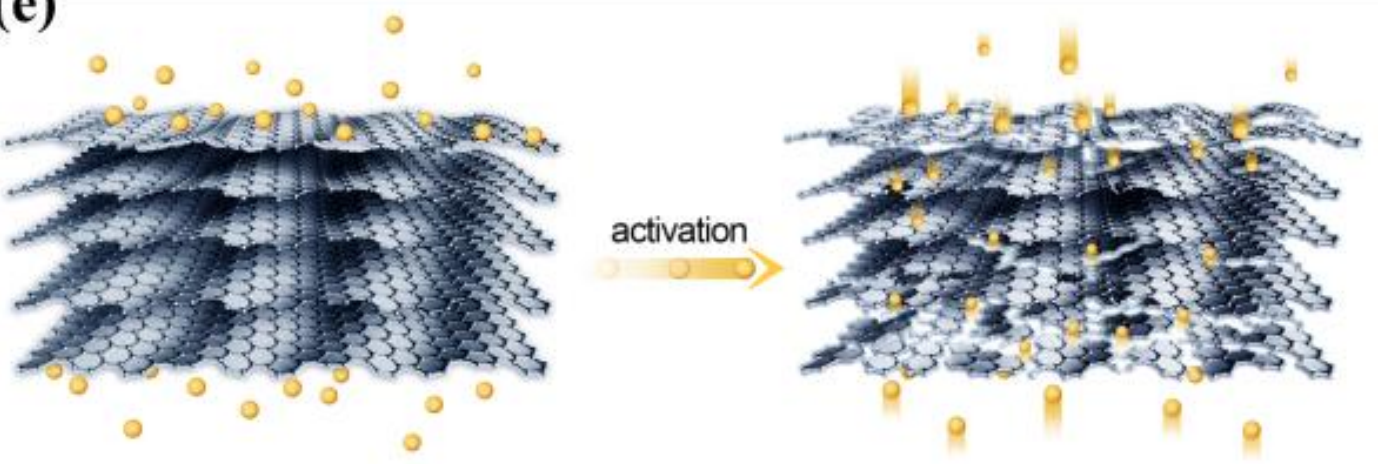

Fig.1 Morphology of the surfaces of the as-prepared samples: (a) AC-0, (b) AC-1, (c) AC-2, and (d) AC-3; scale bars: $100 \mathrm{~nm}$. (e) Schematic illustration of the evolution of the surface on the graphite. As the adsorption of $\mathrm{K}^{+}$ions on the graphite proceeded, the etching reaction was triggered by the following heat-treatment. Finally, the smooth surface was broken and the rough morphology formed on the surfaces of the resultant carbon particles.

The XRD patterns of the as-prepared carbon samples are shown in Fig. 2a. The $2 \theta$ diffraction peaks at $26.6^{\circ}$ and $55^{\circ}$, corresponds to the characteristic peaks of graphite for the (002) and (004) graphite lattice planes, respectively [8]. As shown in Fig. 2b and c, for samples from AC-0 to AC-3, it can be observed that the position of the (002) peak experiences a slight shift to lower diffraction angles, indicating a small change in 
the $d$-spacing. The refined $d$-spacing values are presented in Fig. $2 d$ and indicate that the $d$-spacing experiences an expansion from $0.334 \mathrm{~nm}(\mathrm{AC}-0)$ to around $0.335 \mathrm{~nm}$ (AC-2 and 3), which is close to the $d$-spacing values of reported soft carbon.[9]. Moreover, Fig. 2e shows the d-spacing values of the part formed carbon nanosheets can reach around $0.358 \mathrm{~nm}$. The transmission electron microscope (TEM) images in Fig. $2 \mathrm{f}$ and $\mathrm{g}$ further demonstrate that the activated edges of the graphite flakes have split level or inflated layers. These will benefit the intercalation/deintercaltion of the sodium ions or potassium ions. 

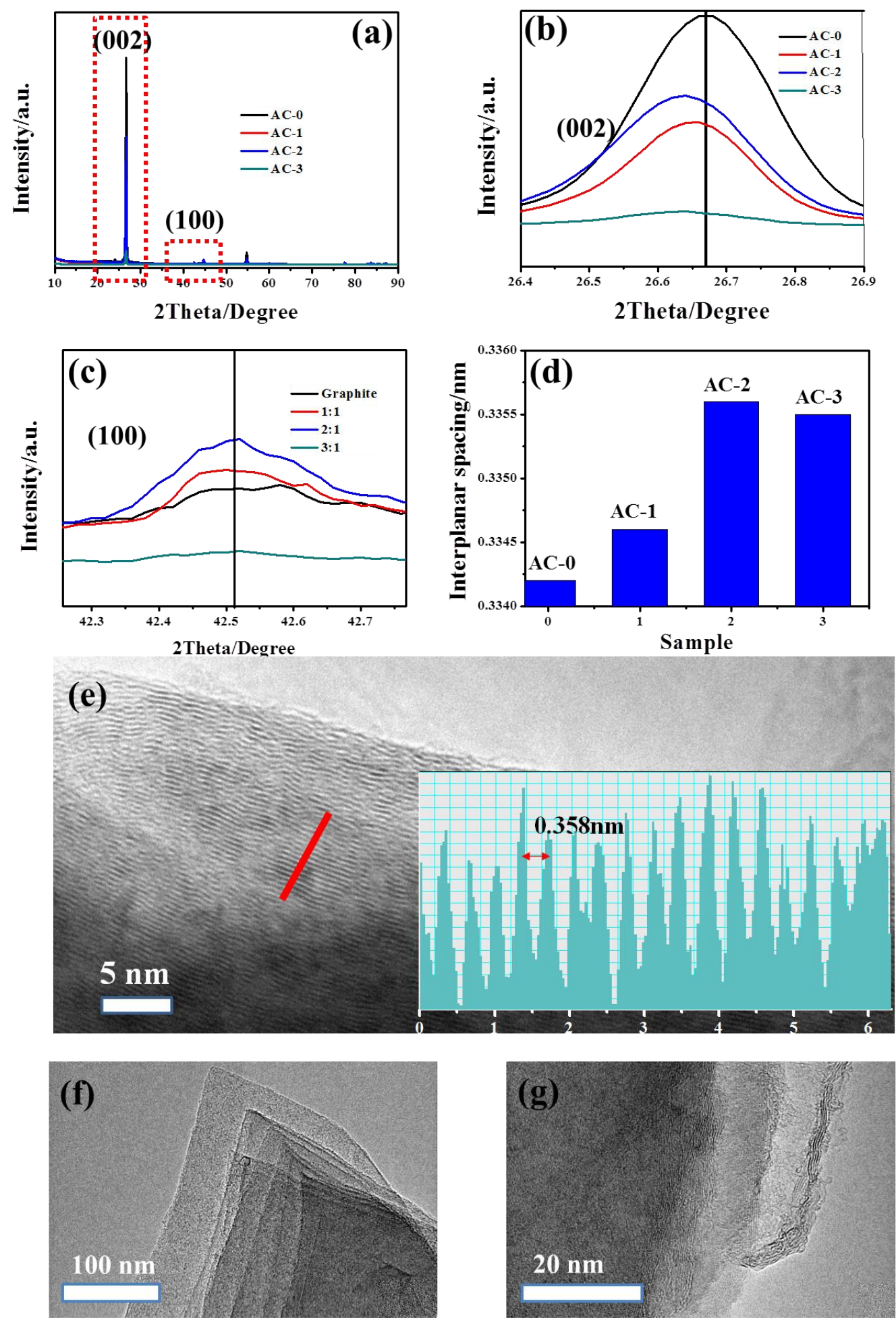

Fig. 2 Structure of the as-prepared samples: (a) XRD patterns, and enlargements of (b) the (002) peak and (c) the (004) peak, respectively. (d) The calculated spacing of the graphite layers in the as-prepared samples. After activation, TEM (e) shows the d-spacing values of the formed carbon nanosheets can reach $\sim 0.358 \mathrm{~nm}$. And TEM images (f) and (g) of AC-2 under different magnifications shows split level or inflated layers. 
To reveal the structural evolution of the graphite samples with increasing amounts of $\mathrm{KOH}$ in the activation process, Raman spectra were collected. In Fig. 3, the G and D bands appear around 1580 and $1350 \mathrm{~cm}^{-1}$, respectively [10]. The $\mathrm{G}$ band corresponds to the $\mathrm{E}_{2 \mathrm{~g}}$ phonon at the Brillouin zone center, and Fig. 3a shows a doubly-degenerate in-plane $s p^{2}$ C-C stretching mode [11]. The D band, which originates from a defect-activated one-phonon double resonance process between the $\mathrm{K}-\mathrm{K}^{\prime}$ symmetry points, reflects the breathing modes of six-atom rings and requires a defect for its activation, as shown in Fig. $3 \mathrm{~b}$ [12]. Therefore, the $\mathrm{D}$ band is also attributed to an $\mathrm{A}_{1 \mathrm{~g}}$ zone-boundary mode of the graphite. Fig. $3 \mathrm{c}$ reveals that all the samples have a strong $\mathrm{G}$ band and a relatively weak $\mathrm{D}$ band. During the activation process, however, the shift in the $\mathrm{G}$ band shows a high dependence on the amount of $\mathrm{KOH}$, and the upshifting trend agrees with a previous report on graphene, where it is demonstrated to be a function of doping [13]. The disorder-induced Raman frequencies can be related to the six dispersive phonon energy branches induced by the activation of the etching process in graphite, which results in the disorder-induced one-phonon peak ( $\mathrm{D}^{\prime}$ band) in the Raman spectra [14]. Since the Raman intensity is proportional to the corresponding vibrational mode, the intensity ratio of the bands, $I_{\mathrm{D}} / I_{\mathrm{G}}$, as shown in Table 1, could be used to evaluate the degree of disorder of the graphite materials. Here, $I_{\mathrm{D}} / I_{\mathrm{G}}$ of AC-2 is around 0.43 , which is higher than for previously published samples [15]. Meanwhile, AC-2 also shows a higher specific surface area than the other samples as the amount of $\mathrm{KOH}$ increases. 
(a)

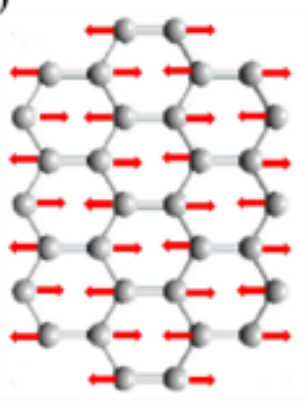

(b)

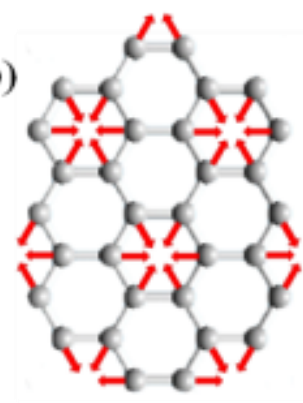

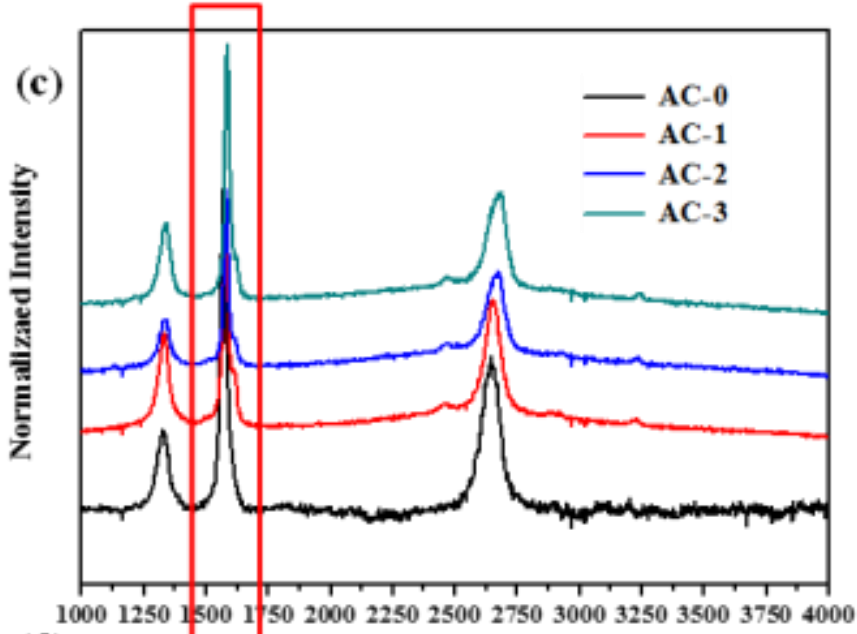

(d)

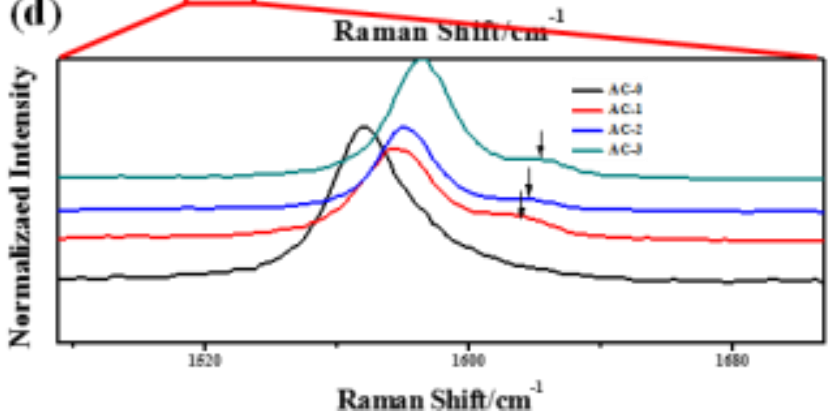

Fig. 3 Illustration of (a) the $E_{2 g}$ vibrational mode of carbon atoms in one graphite layer and (b) an $\mathrm{A}_{\lg }$ zone-boundary mode at the edge of the graphite layer. (c) Raman spectra of the samples obtained at the $532 \mathrm{~nm}$ wavelength, and (d) enlargement of the $\mathrm{G}$ band and $\mathrm{D}^{\prime}$ band region, with the $\mathrm{D}^{\prime}$ band indicated by the arrows.

Table 1 The characteristics of the as-prepared powders. BET is Brunauer-Emmett-Teller.

\begin{tabular}{c|cc}
\hline Sample & $\boldsymbol{I}_{\boldsymbol{D}} / \boldsymbol{I}_{\boldsymbol{G}}$ & BET surface area $/ \mathbf{m}^{2} / \boldsymbol{g}$ \\
\hline$A C-0$ & 0.28 & 3.19 \\
$A C-1$ & 0.32 & 3.77 \\
$A C-2$ & 0.43 & 6.17 \\
$A C-3$ & 0.35 & 5.00 \\
\hline
\end{tabular}



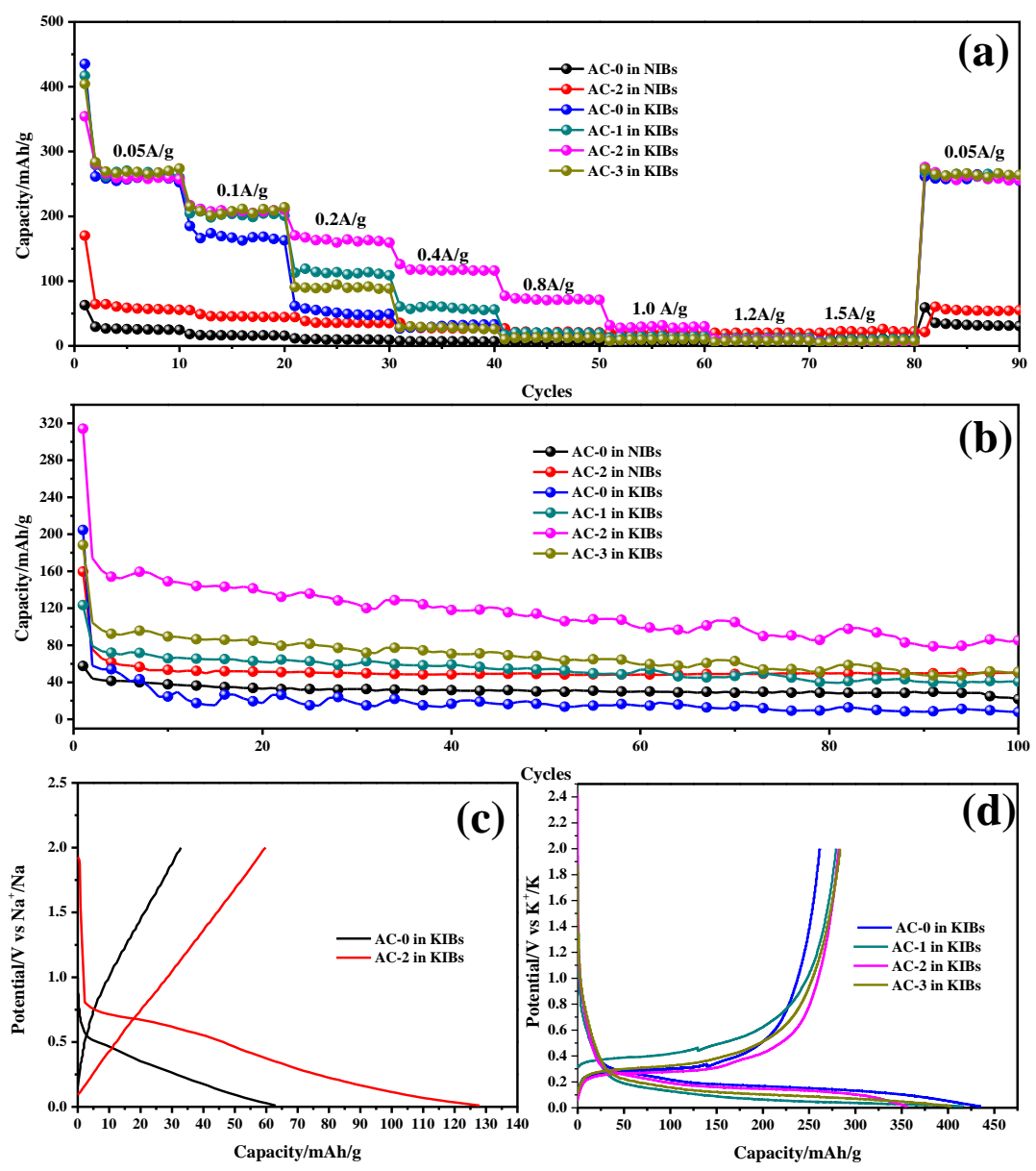

Fig. 4 Electrochemical performance of activated graphite and commercial graphite electrodes: (a) Rate performance and (b) cycling performance of the sample electrodes at $0.2 \mathrm{~A} \mathrm{~g}^{-1}$. (c) $1^{\text {st }}$ cycle discharge/charge curves in the sodium ions battery at a current density of $0.2 \mathrm{~A} \mathrm{~g}^{-1}$ for AC-0 and AC-2 electrodes. (d) $1^{\text {st }}$ cycle discharge/charge curves in the potassium ions battery at a current density of $0.05 \mathrm{~A} \mathrm{~g}^{-1}$ for activated graphite and commercial graphite electrodes.

The activated carbon electrode exhibits greatly enhanced rate capability. Fig. 4a presents successive sequence tests at various current densities from $0.05 \mathrm{Ag}^{-1}$ to 1.5 $\mathrm{Ag}^{-1}$ in NIBs and KIBs. Due to the thermodynamic instability of binary Na-intercalated graphite, graphite has not been considered suitable for the $\mathrm{Na}^{+}$ion battery. The treated graphite of AC-2, however, could exhibit improved capacity compared with the AC-0 untreated graphite, suggesting that activated carbon electrodes could be used for improving the rate capability of the $\mathrm{Na}^{+}$ions battery. The insignificant decay in capacity from the $2^{\text {nd }}$ to the $100^{\text {th }}$ cycle of $\mathrm{AC}-2$ in NIBs 
demonstrates its good stable cycling performance. The galvanostatic discharge/charge curves in Fig. 4c reveal thatAC-2 has a raised dimple and lower-voltage plateau at the beginning of discharge, compared with AC-0. As the dimple and the lower-voltage plateau suggest that the $\mathrm{Na}^{+}$ions need to go through a barrier, which is related to desolvation or solvation shell distortion, it is suggested that the activated graphite in AC-2 has a relatively low barrier for $\mathrm{Na}$ ion intercalation. [14]. In the case of KIBs, as the current density is set higher, the capacity of AC-2 in Fig. 4a overtakes those of $\mathrm{AC}-0,1$, and 3 in KIBs, where the AC-2 shows 209, 159, 114, 72, and $30 \mathrm{mAh} \mathrm{g}^{-1}$ at $0.1,0.2,0.4,0.8$, and $1 \mathrm{~A} \mathrm{~g}^{-1}$, respectively, while AC-0 exhibits 162, 56, 33, 16, and 8 $\mathrm{mAh} / \mathrm{g}$ at the same current densities. The better rate performance of AC-2 may be due to its architecture, consisting of nanosized carbon sheets with larger $d$-spacing in the (100) crystal planes as a result of the etching treatment.

At the relatively high current density of $0.2 \mathrm{~A} \mathrm{~g}^{-1}, \mathrm{AC}-2$ demonstrates a reversible specific capacity of $100.3 \mathrm{mAh} \mathrm{g}^{-1}$ after 100 cycles, which is higher than for the other activated carbon samples. In Fig. 4d, it is shown that the treated electrodes exhibit almost the same high initial discharge capacity and relatively low charge capacity at a current density of $0.05 \mathrm{~A} \mathrm{~g} \mathrm{~g}^{-1}$, and, in particular, that the value of the charge capacity is around $260 \mathrm{mAh} \mathrm{g}^{-1}$, which is close to the theoretical capacity of $279 \mathrm{mAh} \mathrm{g}^{-1}$ for fully intercalated $\mathrm{KC}_{8}$. The higher irreversible capacity is partially attributed to the initial solid electrolyte interphase (SEI) formation and electrolyte decomposition. The reversible discharge plateau ranges from 0.4 to $0.2 \mathrm{~V}$, which is also consistent with the direct formation of $\mathrm{KC}_{8}[2,3]$.

Here, the different values of the discharge plateau of AC-0, AC-1, AC-2 and AC-3 can be attributed to the residual oxygen-containing groups in the samples (as shown in Fig. S1, S2 and Table S1), which would lead to the sloping profile.[16,17] Moreover, there is a huge difference for sodium and potassium electrochemical process after comparing the discharge-charge curves of the AC-0 and AC-2. Graphite electrode has a low capacity for $\mathrm{Na}$ but a high capacity for $\mathrm{K}$. The reasons are still not clearly, however, the possible and reasonable explanation is the formation energy $\left(\mathrm{E}_{f}\right)$ of M-graphite (M=Li, Na, K, Rb, and Cs.) compounds follows the order: 
$\mathrm{Na}>\mathrm{Li}>\mathrm{K}>\mathrm{Rb}>\mathrm{Cs}$, where all $\mathrm{Ms}$ except the $\mathrm{Na}$ have negative $\mathrm{E}_{f}$ with graphite. This means the Na-graphite compounds with high $\mathrm{Na}$ contents are not thermodynamically stable, comparing with other four M-graphite compounds, in agreement with the experimentally observed low Na capacity.[18]
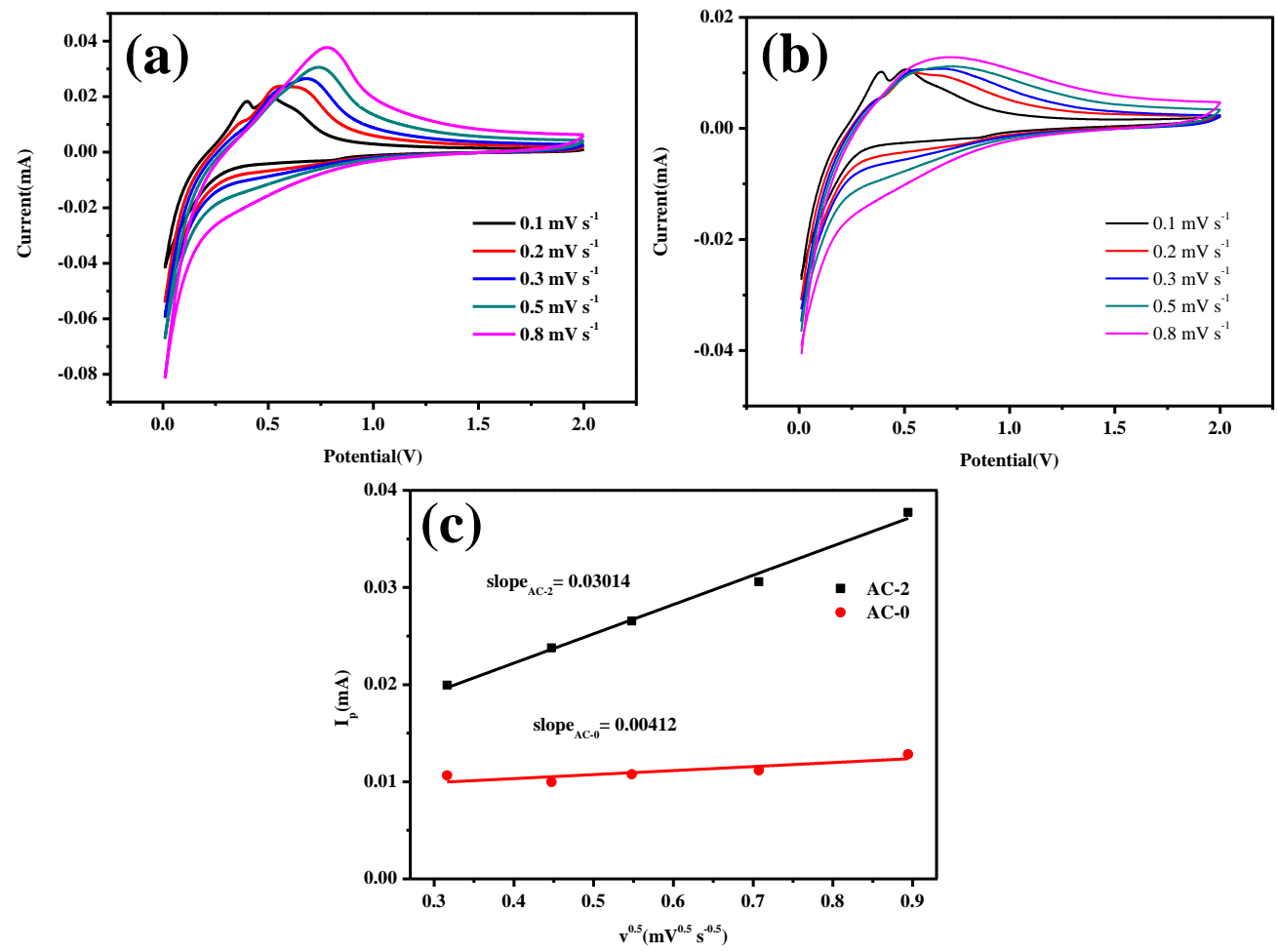

Fig. $5 \mathrm{CV}$ curves at different scan rates of activated graphite and graphite electrodes: (a) AC-2 and (b) AC-0. (c) Peak current versus $m V^{0.5} s^{-0.5}$ and the corresponding linear fits.

The markedly improved electrochemical performance of the activated graphite electrode, compared with the non-activated graphite electrode, was found to be closely associated with $\mathrm{K}$ ion transport in the graphite-based electrode. Cyclic voltammetry $(\mathrm{CV})$ analysis of $\mathrm{AC}-0$ and $\mathrm{AC}-2$ was carried out to evaluate their $\mathrm{K}$ ion diffusion coefficients $(D)$ based on the Randles-Sevcik equation [19]:

$$
I p=0.4463 n F A C \sqrt{\frac{n v F D}{R T}}
$$

Where $I_{p}$ is the peak current, $n$ is the number of electrons, $F$ is the Faraday constant, $R$ 
is the gas constant, $T$ is the temperature, $A$ is the surface area of the electrode, $D$ is the diffusion coefficient, $C$ represents the concentration of potassium ions in the electrolyte, and $v$ stands for the voltage scanning rate. The $\mathrm{K}^{+}$diffusion coefficients in activated graphite and graphite electrodes are calculated from the slope of the plot of $I_{p}$ versus $V^{0.5}$, as shown in Fig. 5c. From the linear relationship between $I_{p}$ and $V^{0.5}$, $D_{\mathrm{AC}-0}$ (hereafter $\mathrm{C}_{0}$, cathodic peak at $\sim 0.52 \mathrm{~V}$ ) and $D_{\mathrm{AC}-2}$ (hereafter $C_{0}$, cathodic peak at $\sim 0.51 \mathrm{~V}$ ) were obtained, and the other parameters were determined: $n=0.125, F=$ $96485.3 \mathrm{C} \mathrm{mol}^{-1}, R=8.314 \mathrm{~J} \mathrm{~mol}^{-1} \mathrm{~K}^{-1}, T=298 \mathrm{~K}, A=0.49 \mathrm{~cm}^{2}$, and $C=0.8 \mathrm{~mol}$ $\mathrm{L}^{-1}$. Among the $\mathrm{K}^{+}$diffusion coefficients, the one for activated carbon $\left(D_{\mathrm{AC}-2}\right)$ was up to $\sim 7$ times larger than for the unactivated graphite $\left(D_{\mathrm{AC}-0}\right)$. This suggests that the surface carbon particles of AC-2 had been smashed into interconnected small porous pieces by the activation treatment, which could not only realize fast electron transfer, but also could facilitate the penetration and diffusion of the electrolyte.

\section{Conclusion}

In conclusion, activated carbon electrodes were developed for the potassium ion battery and fabricated via high-temperature annealing with $\mathrm{KOH}$ as the etching agent. The as-prepared activated carbon with a larger $d$-spacing in the (100) crystal planes allows rapid intercalation/de-intercalation of potassium ions into/from the carbon, and its $\mathrm{K}^{+}$ion diffusion coefficient was also improved by around 7 times compared with the unactivated graphite during the discharge/charge process, contributing to the enhanced electrochemical performance. In particular, the AC-2 electrode showed a rate retention of $54 \%$ as the current density increased from $0.05 \mathrm{~A} \mathrm{~g}^{-1}$ to $1.0 \mathrm{~A} \mathrm{~g}^{-1}$. The results suggest that activated carbon could be a promising anode for the potassium ion battery.

\section{Acknowledgements}

The authors are grateful for financial support from the Auto CRC 2020 project, an ARC Discovery Project (DP170102406), and the China Scholarship Council (CSC). This research used equipment funded by an Australian Research Council (ARC) Linkage, Infrastructure, Equipment and Facilities (LIEF) grant (LE0237478), with the 
facilities located at the UOW Electron Microscopy Centre. The authors greatly appreciate the insightful comments and suggestions provided by Prof. Zaiping Guo. Many thanks are owed to Dr. Tania Silver for critical reading of the manuscript.

\section{References}

[1] Y. Wen, K. He, Y. Zhu, F. Han, Y. Xu, I. Matsuda, Y. Ishii, J. Cumings, C. Wang, Expanded graphite as superior anode for sodium-ion batteries, Nature Communications 5 (2014) 4033.

[2] Z. Jian, W. Luo, X. Ji, Carbon Electrodes for K-Ion Batteries, Journal of the American Chemical Society 137(36) (2015) 11566-9.

[3] S. Komala, T. Hasegawa, M. Dahbi, K. Kubota, Potassiumintercalation into graphite to realize high-voltage/high-power potassium-ion batteries and potassium-ion capacitors, Electrochemistry Communications, 60 (2015) 172-175.

[4] Z. Jian, Z. Xing, C. Bommier, Z. Li, X. Ji, Hard Carbon Microspheres: Potassium-Ion Anode Versus Sodium-Ion Anode, Advanced Energy Materials 6(3) (2016) 1501874.

[5] W. Luo, J. Wan, B. Ozdemir, W. Bao, Y. Chen, J. Dai, H. Lin, Y. Xu, F. Gu, V. Barone, L. Hu, Potassium Ion Batteries with Graphitic Materials, Nano Letters 15(11) (2015) 7671-7.

[6] M.Y. Han, B. Ozyilmaz, Y. Zhang, P. Kim, Energy band-gap engineering of graphene nanoribbons, Physical Review Letters 98(20) (2007) 206805.

[7] X. Wang, H. Dai, Etching and narrowing of graphene from the edges, Nature Chemistry 2(8) (2010) $661-5$.

[8] M.S Sevilla, A.B. Fuertes, Direct Synthesis of Highly Porous Interconnected Carbon Nanosheets and Their Application as High-Performance Supercapacitors, ACS Nano 8(5) (2014) 5060-78.

[9] S. Qiu, Y. Cao, X. Ai, H. Yang. Disscussion on the mechanism of sodium storage of different structure types of cabon materials. Sci Sin Chim, 2017, 47, doi:10.1360/N32016-00236.

[10] P.H.T. Weijie Zhao, Jian Liu, Andrea C. Ferrari, Intercalation of Few-Layer Graphite Flakes with $\mathrm{FeCl}_{3}$ : Raman Determination of Fermi Level, Layer by Layer Decoupling, and Stability, Journal of the American Chemical Society 133 (2011) 6.

[11] F. Tuinstra,.J.L. Koenig, Raman spectrum of graphite, The Journal of Chemical Physics 53 (1970) 1126.

[12] C. Thomsen,.S. Reich, Double Resonant Raman Scattering in Graphite, Physical Review Letter 85 (2000) 5214. 
[13] Z. Ju, S. Zhang, Z. Xing, Q. Zhuang, Y. Qiang, Y. Qian, Direct Synthesis of Few-Layer F-Doped Graphene Foam and Its Lithium/Potassium Storage Properties, ACS Appl Mater Interfaces 8(32) (2016) 20682-90.

[14] M.S. Dresselhaus, A. Jorio, A.G. Souza Filho, R. Saito, Defect characterization in graphene and carbon nanotubes using Raman spectroscopy, Philosophical Transactions. Series A, Mathematical, Physical, and Engineering sciences 368(1932) (2010) 5355-77.

[15] A.C. Ferrari, J. Robertson, Interpretation of Raman spectra of disordered and amorphous carbon, Physical Review B 61 (2000) 14095.

[16] S. Wei, S. Xu, A. Agrawral, S. Choudhury, Y. Lu, Z. Tu, L. Ma, L.A. Archer, A stable room-temperature sodium-sulfur battery, Nature Communications 7 (2016) 11722.

[17] Y.Y. Shao, J. Xiao, W. Wang, M. Engelhard, X.L. Chen, Z.M. Nie, M. Gu, L.V. Saraf, G. Exarhos, J.G. Zhang, J. Liu, Surface-driven sodium ion energy storage in nanocellular carbon foams. Nano Lett. 13 (2013) 3909-3914.

[18] Y.Y. Liu, B.V. Merinov, W.A. Goddard, Origin of low sodium capacity in graphite and generally weak substrate binding of $\mathrm{Na}$ and $\mathrm{Mg}$ among alkali and alkaline earth metals, Proceedings of the National Academy of Sciences, 113(14) (2016) 3735-3739.

[19] H. Wang, W. Zhang, H. Liu, Z. Guo, A Strategy for Configuration of an Integrated Flexible Sulfur Cathode for High-Performance Lithium-Sulfur Batteries, Angewandte Chemie 55(12) (2016) 3992-6.

\section{Supporting Information}

\section{Activated carbon from the graphite with increased rate} capability for the potassium ion battery 
Zhixin Tai, ${ }^{1}$ Qing Zhang, ${ }^{1}$ Yajie Liu, Huakun $\mathrm{Liu}^{2}$ and Shixue Dou

Institute for Superconducting and Electronic Materials, Australian Institute for Innovative Materials, University of Wollongong, Innovation Campus, North Wollongong, New South Wales 2500, Australia

${ }^{1}$ These authors contributed equally to this work.

2 *Corresponding author. Institute for Superconducting and Electronic Materials, Australian Institute for Innovative Materials, University of Wollongong, Innovation Campus,North Wollongong, New South Wales 2500, Australia. Tel:+61242214547 E-mail: hua liu@uow.edu.au 


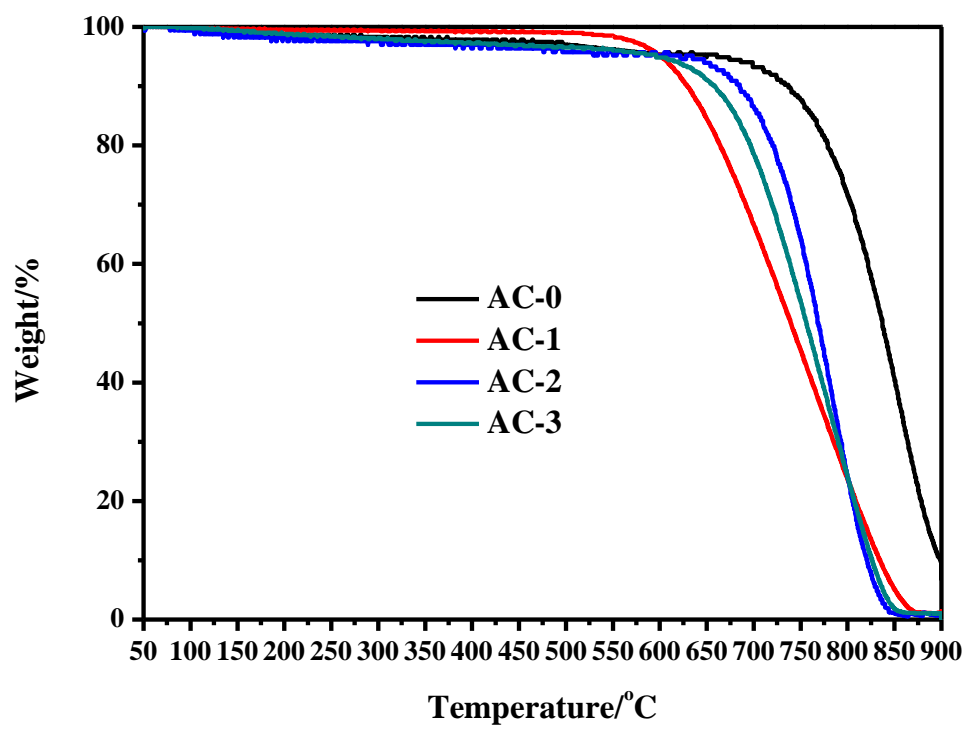

Figure S1 TGA curves of the AC-0, AC-1, AC-2 and AC-3.

To evaluate the amount of the residual potassium in our samples, the TGA was carried out to calculate the amount of potassium as shown in Figure S3. The AC-1, 2 and 3 have shown the similar curves. From the weight loss of the samples, it can be concluded that the amount of the potassium remains in the graphite is no more than $1 \%$. 
Table S1 The percentage of the elements in samples.

\begin{tabular}{cccc}
\hline \hline Items & C1s/wt\% & O1s/wt\% & N1s/wt\% \\
\hline AC-0 & 94.23 & 5.77 & 0.01 \\
AC-1 & 93.29 & 4.44 & 2.26 \\
AC-2 & 93.68 & 6.29 & 0.04 \\
AC-3 & 93.72 & 4.18 & 2.10 \\
\hline
\end{tabular}
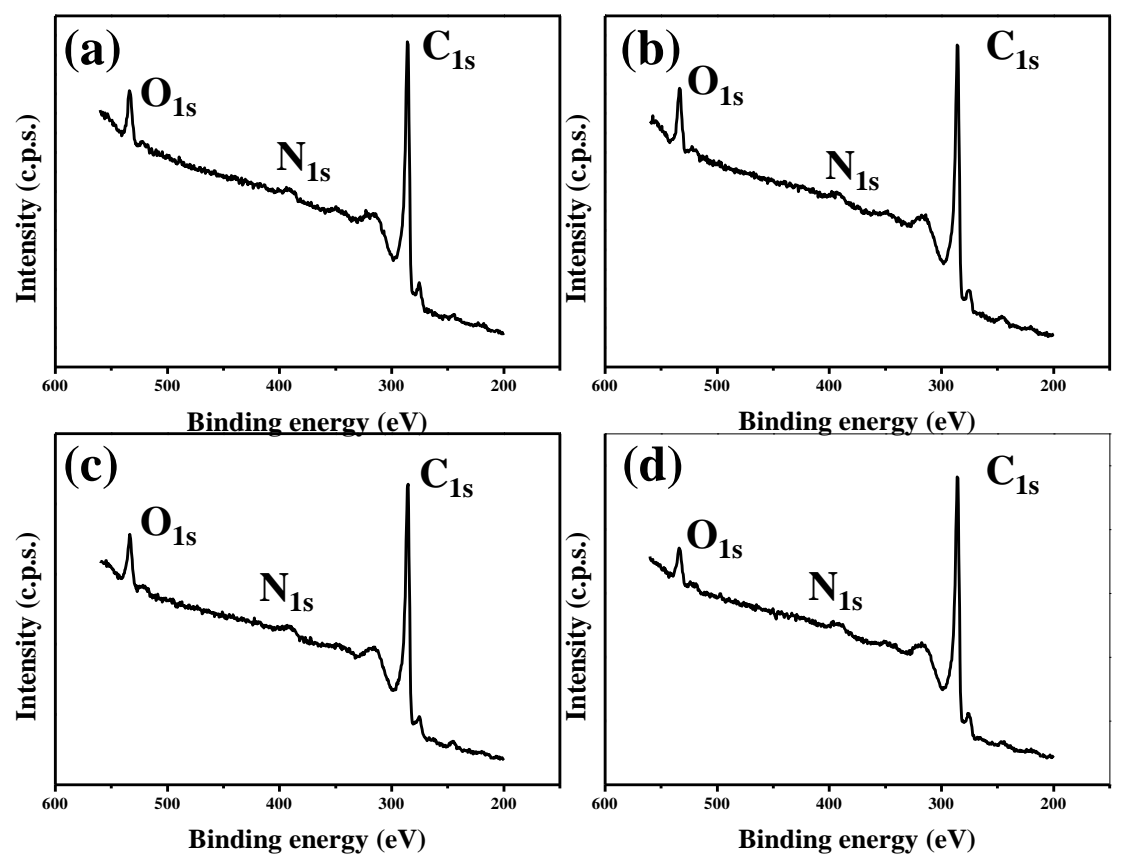

Figure S2 XPS investigation of the carbon, oxygen and nitrogen.(a) AC-0, (b) AC-1, (c) AC-2 and (d) AC-3.

To further confirm whether there are some potassium remains in our samples, XPS was used to confirm the percentage of the elements in the samples. As shown in Table $\mathrm{S} 1$ and Figure $\mathrm{S} 2$, the samples do not have $\mathrm{K}$ but some $\mathrm{C}, \mathrm{O}$ and $\mathrm{N}$. 


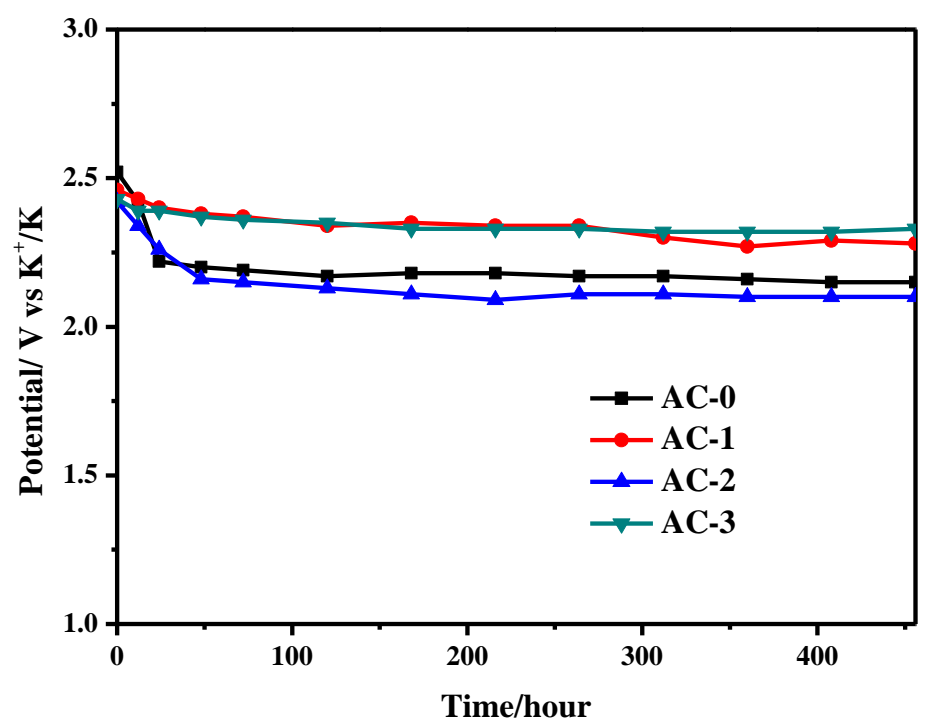

Figure S3 Self-discharge curves of the samples.

The self-discharge shows that the open circuit potential of electrodes would decrease firstly and then keep stable as the time goes on. Here, the AC-0 and AC-2 have a large decrease, which can be attributed to the more side reactions during the standing. 